\title{
Experimental Study of Using a 3-D Position Sensitive CdZnTe Detector for Proton Beam Range Verification
}

\author{
Valerie E. Nwadeyi, Student Member, IEEE, Paul Maggi, Jerimy C. Polf, and Zhong He, Senior Member, IEEE
}

\begin{abstract}
Position sensitive CdZnTe Compton imaging cameras are currently being studied for their use of proton beam range verification for radiotherapy applications. This work presents the use of an experimental large volume CdZnTe detector for the detection of prompt gamma rays that are emitted from protonnuclei interaction within plastic $\left(\mathrm{C}_{2} \mathrm{H}_{4}\right)$ targets. Two experiments were conducted where the incident angle and the dose profile of the beam were varied. The energy spectra from these experiments show that the angle at which the beam enters the target can influence the photopeak to Compton continuum ratios, resulting in more than $18 \%$ increase at $718 \mathrm{keV}$ when the beam is parallel to the detector. Images of the $718 \mathrm{keV}$ and $4.44 \mathrm{MeV}$ characteristic prompt gamma ray emission from carbon-proton interactions are reconstructed using list-mode maximum likelihood expectation maximization (MLEM). Images from these prompt gamma emissions line up well with the expected location of the proton beam within the plastic targets.
\end{abstract}

\section{INTRODUCTION}

$\mathrm{T}$ The use of heavy charged particles, such as protons, in irradiating beams for radiotherapy is known as hadron therapy, and is becoming a sought after therapy for localized cancers. In comparison to photon therapy, proton therapy has the capability of sparing nearby critical organs due to its unique interaction physics and localized energy deposition within the target [1]. In proton radiotherapy, uncertainty arises when determining the exact position of the Bragg peak, where the proton deposits most of its dose via ionization. Using the characteristic prompt gamma rays emitted during proton radiotherapy, a technique for non-invasive monitoring can be established, where the overarching goal is real-time dose monitoring. This relies on the quick detection and analysis of the signature photons emitted during irradiation, with the aim to readapt the planned treatment if the dose is mislocated [2].

Current research is studying the feasibility of proton beam range verification and monitoring via analyzing the prompt gamma-ray signal [3] [4]. Imaging these photons could provide valuable information about the dose profile of the beam and the endpoint within the target [5] [6] [7] [8]. However, there are challenges from the noise associated with their emission. Not only is a high flux of prompt gamma rays emitted, these photons also vary significantly in energy, up to approximately $15 \mathrm{MeV}$ [9]. The cascade that follows the proton-nuclei interaction includes other forms of radiation besides high-energy prompt gammas, which can lead to noise in the data [10]. This includes coincident positron annihilation photons, neutron emissions, and delayed or non-prompt gammas emitted from the immediate surrounding environment or from the proton inelastic scatter within the target [11].
This work studies the feasibility of using a, $322 \mathrm{~cm}^{3}$ active volume, 3-D position-sensitive CdZnTe detector (CZT) to image high-flux and high-energy photons for better characterization of the proton beam. The resulting energy spectra from these proton beam measurements are analyzed to understand the nature of the signature photons emitted from proton-nuclei interactions in the target and the photopeak to noise ratio.

In this work, we study the irradiation of polyethylene $\left(\mathrm{C}_{2} \mathrm{H}_{4}\right.$, $\rho=0.97 \mathrm{~g} / \mathrm{cm}^{3}$ ) targets by various proton beam scenarios. Listmode maximum likelihood expectation maximization (MLEM) image reconstructions are analyzed to determine the feasibility of using CZT as a tool for Compton imaging of specific prompt gamma rays. Namely the prompt gamma emission from carbon: the $718 \mathrm{keV}$ from the deexcitation of ${ }^{10} \mathrm{~B}$ from the proton induced nuclear spallation reaction on ${ }^{12} \mathrm{C}$ and the $4.44 \mathrm{MeV}$ that can be seen either from the deexcitation of ${ }^{11} \mathrm{~B}$ or ${ }^{12} \mathrm{C}$ are analyzed in this work. This study will, aid in the understanding of CZT to detect and image both high $(>1 \mathrm{MeV})$ and low $(<1$ $\mathrm{MeV}$ ) energy photons for varying proton beam irradiations of tissue-equivalent plastic targets.

\section{PROTON THERAPY SignAL}

High energy protons can penetrate skin and tissue, depositing very little dose along a nearly straight path, until the proton loses enough energy and can only interact with the outer shell electrons of the target via ionization. The point at which the proton rapidly and locally deposits nearly all its energy and dose is referred to as the Bragg peak. The uncertainty in the distal range of the beam delivered during treatment can lead to the beam undershooting or overshooting the tumor, resulting in a geometrical miss of the tumor and the delivery of excessive dose to adjacent healthy tissues and critical organs that could potentially be at risk due to their proximity [12]. To mitigate this limitation of proton radiotherapy, effort has been placed in developing methods to measure the proton beam range during the treatment process based on the information acquired from the photons emitted during proton-nuclei scattering within the target.

\section{A. Bragg Peak Characterization}

Accelerated protons can interact with matter through both elastic and inelastic nuclear interactions. Through these processes, the target nuclei enter an excited state and typically decays through the emission of characteristic gamma rays [13].

As the proton-nuclei interactions occur the proton slows down, until its only mode of interaction is ionization with electrons near the end of its track. This slowing down process 
can be theoretically described as the linear stopping power, $S$, which is defined as

$$
S=-\left(\frac{d E}{d x}\right),
$$

where $d E$, is the differential energy loss along the differential path length, $d x$. Plotting the specific energy loss of the accelerated proton in some known material, is referred to as the Bragg curve. The Bragg peak signifies the differential depth where most of the dose is deposited. At this point the dominant mode of interaction is ionization in this concise location and the proton abruptly comes to rest. The Bragg curve can then be converted to dose and used to display the dose deposited by the proton within the target or patient over its entire trajectory [14].

\section{B. Prompt Gamma Ray Energy Lines}

As the highly energetic proton travels through any given medium, it can undergo four different nuclear reactions with the target nuclei of mass number ( $\mathrm{Z}$ ) and atomic mass (A) leading to reaction products that are left in an excited state:

1. The proton can be captured by the target nuclei, causing $(Z, A) \rightarrow(Z+1, A+1)$.

2. The proton can cause a two-body break up reaction where

$$
(Z, A) \rightarrow(Z 1, A 1)+(Z 2, A 2)
$$

in which the sum of $Z 1$ and $Z 2$ equals $Z$ and the sum of A1 and A 2 equals A.

3. The proton can inelastically scatter with the target nucleus in which,

$$
(Z, A) \rightarrow\left(Z, A^{*}\right)
$$

where the nucleus is now in the excited state and will emit some energy to reach its ground state.

4. The proton can cause spallation reactions which take place at energies above the total binding energy of the target nucleus and can produce three or more reaction products adding up in mass up to $\mathrm{A}+1$.

The excited products of these interactions can decay by emitting gamma rays. These photons can be emitted as both prompt and delayed emissions, ranging in half-lives from orders of magnitude less than picoseconds to minutes post proton interaction [9].

For this work, the proton-induced reactions with carbon are the focus, as the target used is a polyethylene plastic with a composition of $\mathrm{C}_{2} \mathrm{H}_{4}$ and deexcitation gammas are not expected from proton-hydrogen interactions. The nuclear deexcitation gamma-ray lines for proton interactions with ${ }^{12} \mathrm{C}$ are shown in Table 1 and their measured half-life are adapted from Kozlovsky [9].

As seen in Table 1, the expected gamma ray lines range in energies up to $15 \mathrm{MeV}$. The mean life of the ${ }^{12} \mathrm{C}$ reactions range in time from shorter than picoseconds to tens of seconds. The emission of these photons is due to elastic scatter, (p, p') and nuclear spallation, $(\mathrm{p}, \mathrm{x})$, which requires a high amount of energy to be transferred to the nucleus to cause it to fragment.

The $718 \mathrm{keV}, 4.438 / 4.44 \mathrm{MeV}$ gamma-ray lines from proton interactions with ${ }^{12} \mathrm{C}$ are used for MLEM reconstructions to cross-reference the prompt gamma emission with the proton beam Bragg curve profile.
TABLE I

\begin{tabular}{l|ccc}
\hline \hline Line $(\mathrm{MeV})$ & Transition & Reaction & Mean Life (s) \\
\hline 0.718 & ${ }^{10} \mathrm{~B}^{*} 0.718$ & ${ }^{12} \mathrm{C}(\mathrm{p}, \mathrm{x}){ }^{10} \mathrm{~B}^{*}$ & $1.0 \times 10^{-9}$ \\
& ${ }^{12} \mathrm{C}(\mathrm{p}, \mathrm{x}){ }^{10} \mathrm{C}(\mathrm{e}){ }^{10} \mathrm{~B}$ & 27.8 \\
2.000 & ${ }^{11} \mathrm{C}^{*} 2.000$ & ${ }^{12} \mathrm{C}(\mathrm{p}, \mathrm{x}){ }^{11} \mathrm{C}^{*}$ & $1 \times 10^{-14}$ \\
2.124 & ${ }^{11} \mathrm{~B}^{*} 2.125$ & ${ }^{12} \mathrm{C}(\mathrm{p}, \mathrm{x}){ }^{11} \mathrm{~B}^{*}$ & $5.5 \times 10^{-15}$ \\
4.438 & ${ }^{12} \mathrm{C}^{*} 4.439$ & ${ }^{12} \mathrm{C}\left(\mathrm{p}, \mathrm{p}^{\prime}\right)^{12} \mathrm{C}^{*}$ & $6.1 \times 10^{-14}$ \\
4.444 & ${ }^{11} \mathrm{~B}^{*} 4.445$ & ${ }^{12} \mathrm{C}(\mathrm{p}, 2 \mathrm{p}){ }^{11} \mathrm{~B}^{*}$ & $5.6 \times 10^{-19}$ \\
6.337 & ${ }^{11} \mathrm{C}^{*} 6.339$ & ${ }^{12} \mathrm{C}(\mathrm{p}, \mathrm{x}){ }^{11} \mathrm{C}^{*}$ & $<1.1 \times 10^{-13}$ \\
6.476 & ${ }^{11} \mathrm{C}^{*} 6.478$ & ${ }^{12} \mathrm{C}(\mathrm{p}, \mathrm{x}){ }^{11} \mathrm{C}^{*}$ & $<8.7 \times 10^{-15}$ \\
6.741 & ${ }^{11} \mathrm{~B}^{*} 6.743$ & ${ }^{12} \mathrm{C}(\mathrm{p}, \mathrm{x}){ }^{11} \mathrm{~B}^{*}$ & $4.3 \times 10^{-20}$ \\
15.10 & ${ }^{12} \mathrm{C}^{*} 15.11$ & ${ }^{12} \mathrm{C}\left(\mathrm{p}, \mathrm{p}^{\prime}\right){ }_{12} \mathrm{C}^{*}$ & $1.5 \times 10^{-17}$ \\
\hline \hline
\end{tabular}

Expected proton induced reactions on ${ }^{12} \mathrm{C}$ and the corresponding energy line and the resulting transition reaction and mean life.

\section{DETECTION SETUP}

\section{A. Detector System}

Measurements were conducted using the Polaris J3 detector, developed by H3D Inc. (Ann Arbor, MI), at the Maryland Proton Treatment Center (MPTC). This system has two detection stages, each stage contains four modules, with each module consisting of a $2 \times 2$ array of CZT crystals. The modules in the first stage (closest to the proton beam) are comprised of crystals that are $2.0 \mathrm{~cm} \times 2.0 \mathrm{~cm} \times 1.0 \mathrm{~cm}$ and the second stage module (farther from the proton beam) are comprised of crystals that are $2.0 \mathrm{~cm} \times 2.0 \mathrm{~cm} \times 1.5 \mathrm{~cm}$. This results in in 64 total CZT crystals with a total active volume of $322 \mathrm{~cm}^{3}$, the largest detection volume CZT array system built by H3D. Each crystal is equipped with 121 anode pixels and a planar cathode, allowing for 3-D position sensing and energy read out [15].

\section{B. Experimental Setup}

For this study, two different beam experiments were performed on two cylindrical polyethylene $\left(\mathrm{C}_{2} \mathrm{H}_{4}\right)$ targets with a density of $0.97 \mathrm{~g} / \mathrm{cc}$.

The first experiment uses a target with a radius of $8.95 \mathrm{~cm}$ and varies the angle of the beam's entrance into the target. As shown in figure 1 , a single, monoenergetic $120 \mathrm{MeV}$ proton beam was delivered at three different angles: $0^{\circ}$ (vertically), $270^{\circ}$ (horizontally), and $315^{\circ}$ with respect to the floor. This experiment is referred to as the "Beam Angle Dependent" experiment, which is comprised of three measurement angles: "Angle $0^{\circ}$ ", "Angle 270", and "Angle 315".

The second experiment uses a larger target with a radius of $16.95 \mathrm{~cm}$ and focuses on the nature of the Bragg peak profile. For this experiment, the proton beam was altered such that three different Bragg peak profiles were measured. The first measurement consisted of a single $120 \mathrm{MeV}$ pencil beam resulting in a "point" or "spot" like profile. The second measurement uses the same beam energy but magnetically steers the protons in the lateral direction (perpendicular to the 


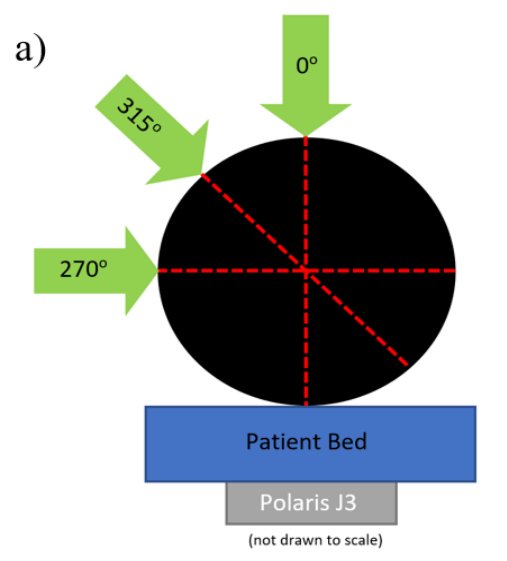

c)

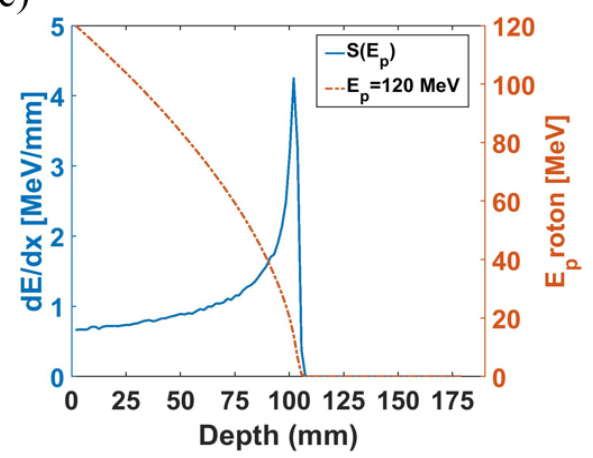

b)

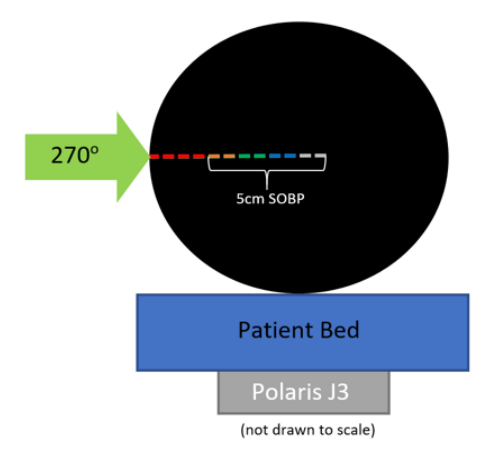

d)

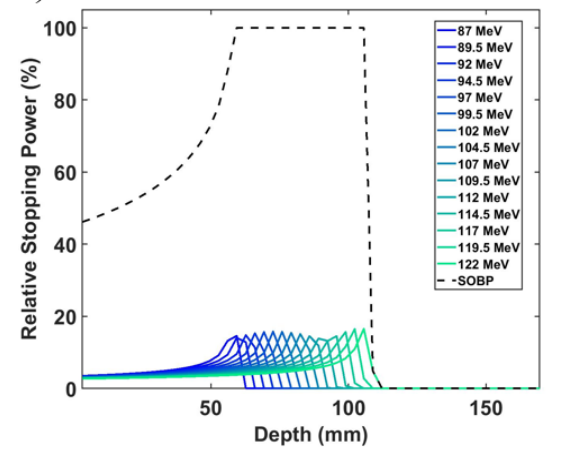

Fig. 1. Schematic of the two experimental setups for proton beam measurements. (a) The Beam Angle Dependent experiment alters the proton beam's incident angle into the target at Angle $0^{\circ}$ (normal), Angle $270^{\circ}$ (parallel), and Angle $315^{\circ}$ with respect to the floor. (b) The Bragg Peak Profile experiment focuses on varying the shape of the Bragg peak profile: $\mathrm{Spot}$, $5 \mathrm{~cm}^{2} \mathrm{Layer}$, $5 \mathrm{~cm}^{3}$ SOBP. (c) The SRIM calculated stopping range for the Beam Angle Dependent measurement is shown as a function of depth and proton energy. (d) The relative stopping power for each energy layer of the SOBP profile for the Bragg Peak Profile experiment.

beam central axis) such that a single $5 \mathrm{~cm}^{2}$ plane of dose is delivered. The third measurement extends the Bragg peak in a $5 \mathrm{~cm}^{3}$ volume within the target. This measurement uses 14 beam energies ranging from $87 \mathrm{MeV}$ to $122 \mathrm{MeV}$ to deliver the $5 \mathrm{~cm}^{3}$ SOBP. This experiment will be referred to as the "Bragg Peak Profile" experiment, which is comprised of three measurements: "Spot", " $5 \mathrm{~cm}^{2}$ Layer", and " $5 \mathrm{~cm}^{3}$ SOBP".

For each measurement, Polaris J3 is attached to the underside of a patient bed, while the phantom is placed on top of the patient bed. Figure 1 shows a schematic of each experimental setup and simulated Bragg curves corresponding to the 120 $\mathrm{MeV}$ beam used in the Beam Angle Dependent experiment and the 14 layers used for the $5 \mathrm{~cm}^{3}$ SOBP measurement. The stopping power simulations are developed using SRIM (Stopping Range of Ions in Matter) [16].

\section{RECORDED PROMPT GAMMA RAY SignAL}

Figure 2 shows a raw count energy spectrum up to $6 \mathrm{MeV}$ to compare each experiment. The notable photopeaks seen in the data are listed in Table 2. The $718 \mathrm{keV}$ peak is the most prominent carbon-proton interaction peak seen and is used for evaluation of the detector's ability to image to the prompt gamma emission. Table 3 shows the Peak to Compton continuum Ratio (PCR) for each measurement for the $718 \mathrm{keV}$ photopeak which is based on the peak area counts in a $10 \mathrm{keV}$ window about the $718 \mathrm{keV}$ peak with respect to the average counts in the continuum to the left and right of the peak within a $10 \mathrm{keV}$ range.

Due to Doppler broadening and the worsened energy resolution of CZT at high photon energies, the $4.44 \mathrm{MeV}$ photopeak is broadened and not as easily resolved as the 718 $\mathrm{keV}$ peak Error! Reference source not found. However, structures of the single and double escape peak can be seen along with a broad shoulder at $4.44 \mathrm{MeV}$, indicating full energy deposition events of the $4.44 \mathrm{MeV}$ gamma ray. Table 3 includes the peak to escape ratio (PER) for the $4.44 \mathrm{MeV}$ peak to show how many events are loss to pair production or that are not fully resolved as photopeak events. The PER is calculated as the peak area counts in a $50 \mathrm{keV}$ window about the $4.44 \mathrm{MeV}$ peak relative to the average of the counts in a $50 \mathrm{keV}$ window about $3.93 \mathrm{MeV}$ and $3.42 \mathrm{MeV}$ corresponding to the single and double escape peaks, respectively.

In both experiments, the presence of neutrons can be denoted by the observed $558 \mathrm{keV}$ peak from thermalized neutrons captured by ${ }^{113} \mathrm{Cd}$ in the detector and the recorded $2.21 \mathrm{MeV}$ peak from the neutron capture on hydrogen in the target. 

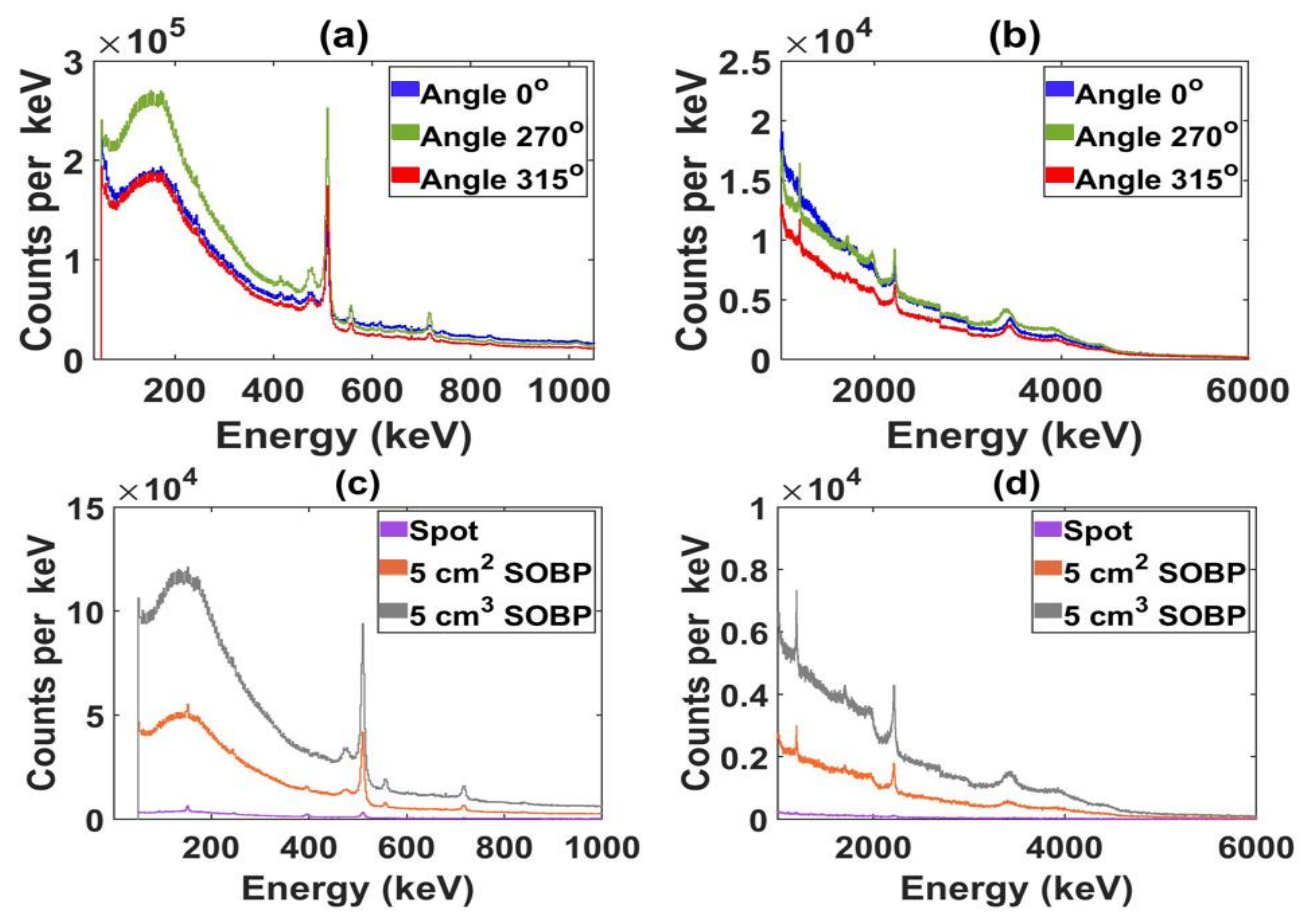

Fig. 2. Energy spectra recorded with Polaris J3 for the (a and b) Beam Angle Dependent experiment and the (c and d) Bragg Peak Profile experiment. Both sets of spectra are shown from 0 to $1000 \mathrm{keV}$ and 1000 to $6000 \mathrm{keV}$.

\begin{tabular}{c|c}
\multicolumn{2}{c}{ TABLE 2 } \\
\hline \hline $\begin{array}{c}\text { Line } \\
(\mathrm{MeV})\end{array}$ & Reaction \\
\hline 0.474 & ${ }^{11} \mathrm{~B}(\mathrm{n}, \alpha)^{7} \mathrm{Li}^{*}$ \\
0.511 & Annihilation photons \\
0.558 & ${ }^{113} \mathrm{Cd}(\mathrm{n}, \gamma)^{114} \mathrm{Cd}^{*}$ \\
0.718 & ${ }^{12} \mathrm{C}(\mathrm{p}, \mathrm{x})^{10} \mathrm{~B}^{*}$ \\
2.221 & ${ }^{1} \mathrm{H}(\mathrm{n}, \gamma)^{2} \mathrm{H}^{*}$ \\
4.438 & ${ }^{12} \mathrm{C}\left(\mathrm{p}, \mathrm{p}^{\prime}\right) 12 \mathrm{C}^{*}$ \\
4.444 & ${ }^{12} \mathrm{C}(\mathrm{p}, 2 \mathrm{p})^{11} \mathrm{~B}^{*}$ \\
\hline \hline
\end{tabular}

Notable recorded gamma ray photopeaks from both the Beam Angle Dependent and Bragg Peak Profile experiments.

\section{A. Beam Angle Dependent Experiment}

The Beam Angle Dependent experiment shows that when the beam is parallel to the detector surface or positioned at $270^{\circ}$, the PCR is improved by more than $18 \%$ for the $718 \mathrm{keV}$ peak in comparison to the Angle $315^{\circ}$ and the Angle $0^{\circ}$ measurements. However, the PER for the $4.44 \mathrm{MeV}$ photopeaks in this experiment are consistent at all three beam angles. This could be because most of the $4.44 \mathrm{MeV}$ emissions are expected to have originated from the end of the proton's track. In this region of the beam the protons are at a very low energy, and based on the cross-section as a function of energy for the ${ }^{12} \mathrm{C}\left(\mathrm{p}, \mathrm{p}^{\prime}\right)^{12} \mathrm{C}^{*}$ and the ${ }^{12} \mathrm{C}(\mathrm{p}, 2 \mathrm{p}){ }^{11} \mathrm{~B}^{*}$ reactions this is when the $4.44 \mathrm{MeV}$ photon is most probable [9-Kozlovsky]. Near the end of the proton beam's track the photon emissions are not as angle or beam trajectory dependent, so its emission site can be treated as an isotropic quasi-point source centralized near the end of the proton track.

Comparing the PER from the $718 \mathrm{keV}$ peak shows that with the beam angled parallel to the detector allows for an improvement in detection of events that happens along the beam's path.

\begin{tabular}{l|ll}
\multicolumn{3}{c}{ TABLE 3 } \\
\multicolumn{1}{c}{ Measurement } & PCR & \multicolumn{1}{c}{ PER } \\
& $718 \mathrm{KeV}$ & \multicolumn{1}{c}{$4.44 \mathrm{MeV}$} \\
\hline Angle $0^{\circ}$ & $0.55 \pm 0.15$ & $0.055 \pm 0.034$ \\
Angle $270^{\circ}$ & $0.69 \pm 0.17$ & $0.052 \pm 0.033$ \\
Angle $315^{\circ}$ & $0.58 \pm 0.21$ & $0.054 \pm 0.036$ \\
\hline Spot & $0.62 \pm 0.41$ & -immeasurable \\
$5 \mathrm{~cm}^{2}$ Layer & $0.64 \pm 0.36$ & --immeasurable \\
$5 \mathrm{~cm}^{3}$ SOBP & $0.63 \pm 0.36$ & $0.053 \pm 0.033$ \\
\hline \hline
\end{tabular}

Photopeak to Compton ratio (PCR) and photopeak to escape peak ratio (PER) calculated for the $718 \mathrm{keV}$ and $4.44 \mathrm{MeV}$ photopeaks, respectively.

\section{B. Bragg Peak Profile Experiment}

In the Spot measurement, using the single $120 \mathrm{MeV}$ beam, very low energy photopeaks at $151 \mathrm{keV}, 200 \mathrm{keV}$, and 396 are seen in the raw results. However, at such low energies, majority of these photopeak events result in photoelectric absorption before scattering in the CZT and cannot be used for Compton imaging. The PER for the $4.44 \mathrm{MeV}$ photopeak could not be measured for both the Spot and $5 \mathrm{~cm}^{2}$ Layer measurements as there were not enough statistics to define a peak in the 4.44 $\mathrm{MeV}$ range of the spectra. The reduction in events in the spectra for the Spot and $5 \mathrm{~cm}^{2}$ SOBP measurements can be due to the short time frame of each measurement compared to that of the $5 \mathrm{~cm}^{3}$ SOBP measurement, which would take longer to irradiate a cubic volume of the target thus allowing the detector more time to record events.

\section{MLEM IMAGING RESULTS}

List-mode maximum likelihood expectation maximization (MLEM) [17] is used to reconstruct $4 \pi$ 2D images of the 718 $\mathrm{keV}$ and $4.44 \mathrm{MeV}$ photopeaks from each measurement of the 
Beam Angle Dependent experiment and for the $5 \mathrm{~cm}^{3}$ SOBP measurement of the Bragg Peak profile experiment. To image the specific photopeaks, all recorded photons that occur in 2, 3, or 4-pixel scatter events are used for Compton imaging [19].

Imaging of the $4.44 \mathrm{MeV}$ photopeak is hindered by the worsened energy resolution of CZT at this energy. At this energy we expect a poorer event reconstruction due to: (1) limited dynamic range of the system up to $3 \mathrm{MeV}$ on the readout AISC for single-pixel events; (2) the timing resolution under such a high flux of photons, and (3) the increase in the number of charge sharing and multiple scattered events which can cause an increase in chance coincidence pixels triggers [20].

To improve the imaging performance of the $4.44 \mathrm{MeV}$ photopeak, Compton events that resulted in a total recorded energy within a $200 \mathrm{keV}$ energy window about the photopeak (between 4.34 to $4.54 \mathrm{MeV}$ ) were accepted as full energy deposition events. FIL-MSD (first is largest mean square difference), developed by Shy and He [21], is used to choose the optimal sequencing of each event and only that Compton cone is used for the MLEM reconstructions. In doing so, poorly reconstructed Compton cones that are incorrectly sequenced are discarded, which would have further hindered the reconstruction of the true source location. Using the optimally reconstructed cone mitigates the need to develop a specific system response function for MLEM to achieve the same result. This would require much computational effort to develop given our submillimeter position resolution on CZT detectors and high-energy deposition requirements. Discarding the Compton cones that are most-likely mis-sequenced aids in minimizing the noise in the imaging space, as the incorrectly sequenced cones of these events will not reconstruct to our true source location or would have more of a Gaussian spread due to its high uncertainty.
The large number of background noise events and Compton continuum, seen in Fig. 2, also contributes to the noise artifacts seen during reconstruction. These events correspond to partial energy deposition events and chance coincidence events that create incorrectly reconstructed Compton cones in the image space. At high photon energies, charge sharing events and pair production events become more likely and lead to incorrect event sequencing for Compton cone reconstruction. This can cause the suppression of the source location within the image space as these events become high frequency noise artifacts that degrades the signal-to-noise ratio (SNR) of the true source location [22].

\section{A. Beam Angle Dependency Experiment}

The same dose rate and beam energy of $120 \mathrm{MeV}$ was used for each beam angle orientation. The expectation is for the beam to stop at the same location within the cylindrical target, near isocenter. According to the SRIM analysis shown in Fig. 1, the distal fall-off of the Bragg peak is approximately $1.55 \mathrm{~cm}$ past isocenter $(\mathrm{y}=0 \mathrm{~cm})$ for a $120 \mathrm{MeV}$ beam impinging a $\mathrm{C}_{2} \mathrm{H}_{4}$ target with a density of $0.97 \mathrm{~g} / \mathrm{cm}^{3}$, corresponding to a full depth of $10.5 \mathrm{~cm}$. In Fig. 3, the expected Bragg peak location (1.23 $\mathrm{cm}$ ) and the entrance point of the beam into the target, with respect to the detectors surface, is marked in each image reconstruction. The image reconstructions show that $718 \mathrm{keV}$ can be used as an estimator of the beam location but is not a good indicator of the beam's Bragg peak. Specifically, when the beam is at Angle $0^{\circ}$, we can see the exact location of the beam, as its trajectory is normal to the center of the detector, but this $2 \mathrm{D}$ reconstruction cannot provide information on the depth of the beam into the target. In comparison of noise artifacts, the Angle $270^{\circ}$ reconstructions outperform the other beam angles as the noise is better suppressed in the imaging space and the entirety of the hotspot is within range of the beam.
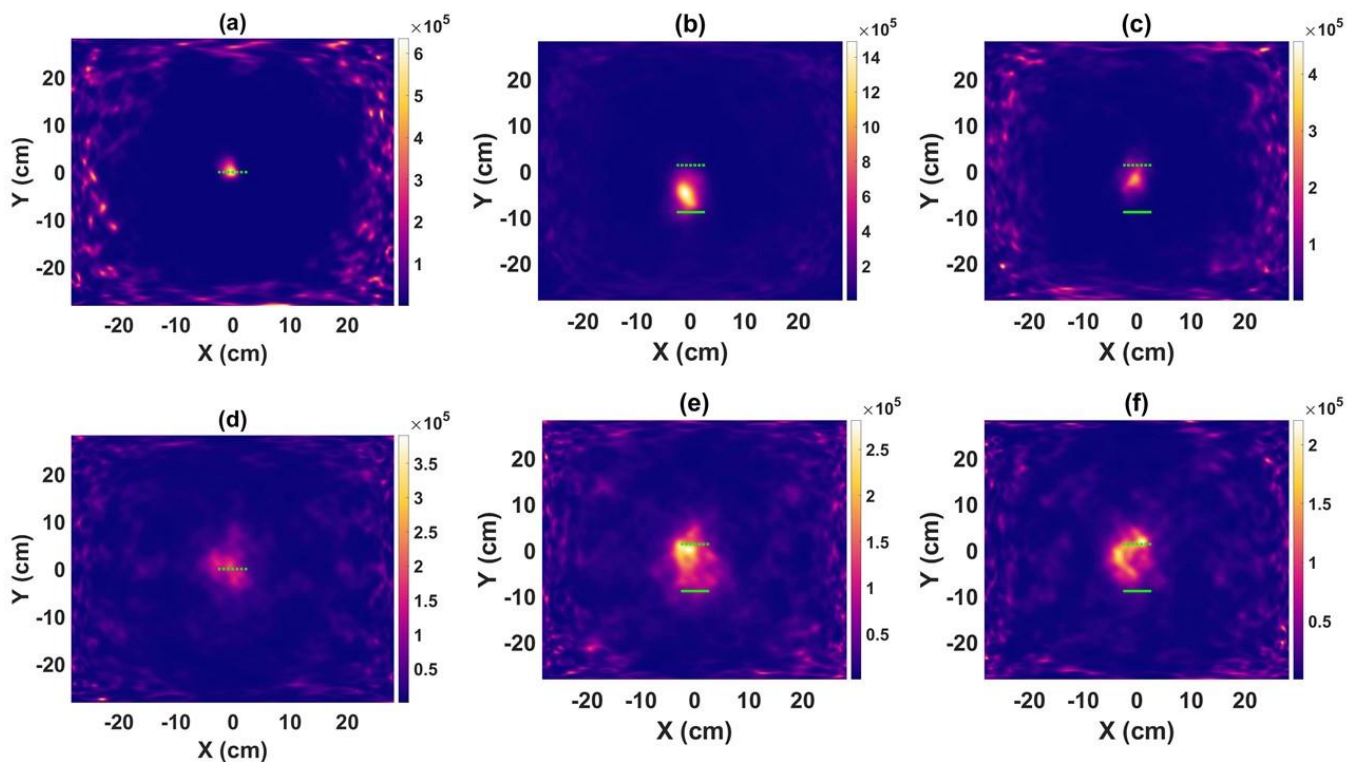

Fig. 3. MLEM image reconstruction of the $718 \mathrm{keV}$ (a-c) and the $4.44 \mathrm{MeV}$ (d-f) photopeaks for the Beam Angle Dependent experiment. Angle $0^{\circ}$ (a and d), Angle $270^{\circ}$ (b and e), and Angle $315^{\circ}$ ( $\mathrm{c}$ and f) The dotted green line represent the expected location of the beam's end point and the solid green line represents the entrance point of the beam into the target, with respect to the 2D imaging space. 

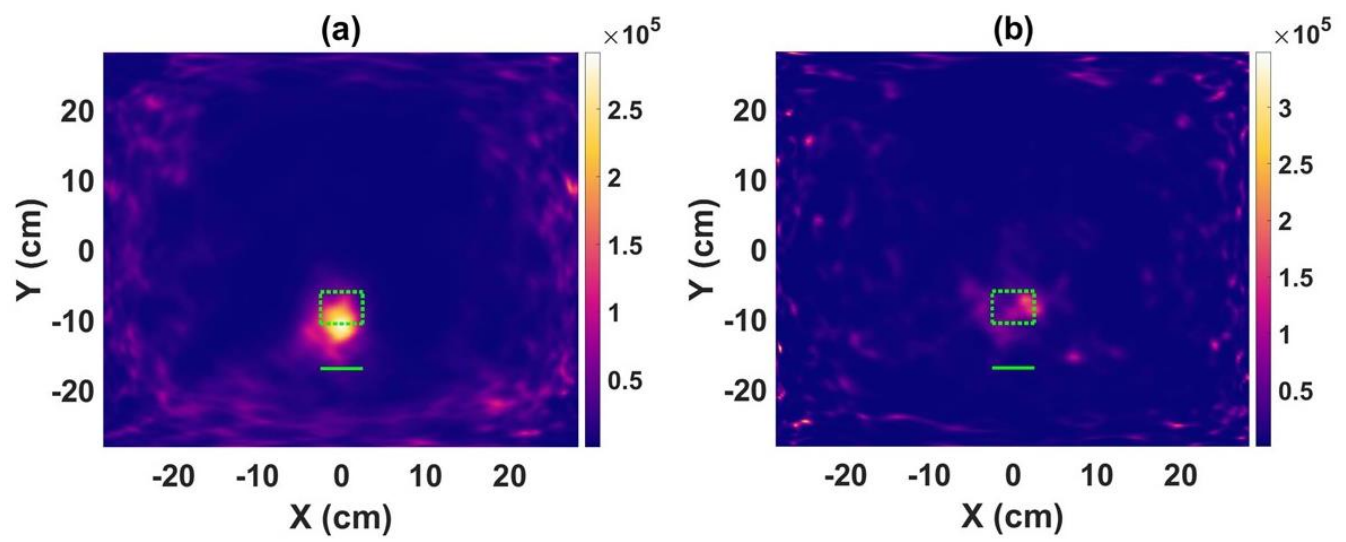

Fig. 4. MLEM reconstructions of the $5 \mathrm{~cm}^{3}$ SOBP measurement from the Bragg Peak Profile experiment. (a) $718 \mathrm{keV}$ image reconstruction and (b) $4.44 \mathrm{MeV}$ image reconstruction. The dashed green box indicates the position and distribution expected of the SOBP in a 2D space $(5 \mathrm{~cm} \times 5 \mathrm{~cm})$ the solid green line represents the entrance point of the beam with respect to the imaging space.

The Angle $315^{\circ}$ suffers from the noise artifacts in the image space, which suppresses the iterative estimate intensity of the true source location. This could be due to the increased attenuation of the events along the angled trajectory of the photons through more of the material before it reaches the detector.

The 4.44 MeV photopeak reconstructions (bottom row of Fig. 3) suffer from the poorer spectral results but show promise of estimating the beams end point despite the noisiness and blur of the hotspot in the final reconstruction. Due to small angle scatters of high energy photons and pair production events, the reconstructed hotspot of the $4.44 \mathrm{MeV}$ photopeak is less concise when compared to the $718 \mathrm{keV}$ reconstruction. However, the Angle $270^{\circ}$ measurement shows that the 4.44 $\mathrm{MeV}$ is more representative of the beam's end point or Bragg Peak location as opposed to the $718 \mathrm{keV}$ peak.

Figure 5a shows a 1D profile of both the $718 \mathrm{keV}$ and the 4.44 MeV image reconstructions for the Angle $270^{\circ}$ measurements. The uncertainty of the FWHM estimation is based on the limit of the pixel resolution which is $0.5 \mathrm{~cm}$. Although the FWHM of the $4.44 \mathrm{MeV}$ profile is much larger than that of the $718 \mathrm{keV}$ profile, it shows that the peak centroid of the $.44 \mathrm{MeV}$ reconstruction is very near the Bragg peak location estimating at $0 \mathrm{~cm}+/-0.5 \mathrm{~cm}$ which is off from the Bragg peak location by $1.23 \mathrm{~cm}$. Whereas the $718 \mathrm{keV}$ peak centroid reconstructs approximately $6.5 \mathrm{~cm}$ away from the Bragg peak. The spread in the FWHM of the $4.44 \mathrm{MeV}$ profile stems from the poorer event reconstruction spectrally which also affects the image reconstruction and adds additional gaussian blur to each cone used for reconstruction. The FWHM and the FWTM for both photopeaks decrease along the X direction or about the width of the proton beam in Figure $5 \mathrm{~b}$, which is measured as a full width of $3.8 \mathrm{~mm}$ based on the expected straggle radially from SRIM simulations. Although there is some suppression in the spread of the image reconstructions in this direction it is still much wider than the expected width of the beam.

\section{B. Bragg Peak Profile Experiment}

For imaging analysis, only the $5 \mathrm{~cm}^{3} \mathrm{SOBP}$ measurement is used as it resulted in enough statistics in both the $718 \mathrm{keV}$ and 4.44 MeV for image reconstructions (see Table 3).
Figure 4 shows the outline of the expected location of the 2D spread of the SOBP for the $5 \mathrm{~cm}^{3}$ SOBP measurement, as indicated by the dotted green line, and the entrance point of the beam into the target represented by the solid green line. In the $718 \mathrm{keV}$ reconstruction the hotspot shows uniformity in its spread as if mimicking the spread of the Bragg peak profile. However, like the Angle $270^{\circ}$ measurement in the Beam Angle Dependent experiment, the hotspot is not a good indicator of the endpoint of the beam, as it does not extend to the final layer of the SOBP. The $4.44 \mathrm{MeV}$ reconstructions suffers from less photopeak events when compared to the Beam Angle Dependent experiment. However, the reconstruction shows that the hotspot location does shift nearer the end of the SOBP proton beam track, following the same findings in the $4.44 \mathrm{MeV}$ reconstructions shown in Figure 2.

FWHM analysis could not be confidently conducted for the 4.44 MEV reconstruction due to the low intensity and sparseness of the hotspot. However, Figure $5 \mathrm{c}$ and $5 \mathrm{~d}$ shows the $718 \mathrm{keV}$ 1D profiles in both the $\mathrm{x}$ and $\mathrm{y}$ direction shows the uniformity of the hotspot which are both estimated at about 5 $\mathrm{cm}$ with approximately a $1 \mathrm{~cm}$ difference. When focusing on the 1D profile along the beam's trajectory, Figure 5c, we see that the peak reconstructs to the location of the first layer of the SOBP at $-10.5 \mathrm{~cm}$ approximately $4.6 \mathrm{~cm}+/-0.5 \mathrm{~cm}$ away from the end of the SOBP.

\section{CONCLUSION}

As seen in all energy spectra presented, the photon emission from proton therapy presents a vast amount of background noise and a large continuum beneath the expected prompt gamma ray lines, all of which can be due to scattered neutrons, partial deposition events from prompt and delayed photons, and background from the room and beam nozzle, and detector limitations such as timing resolution and dynamic range. The Beam Angle Dependent experiment shows that for the same beam current and proton energy, there is a change in the peak ratios depending on the beam's point of entry, which could be leveraged for further analysis when deciding on the geometry used for future beam measurements and for imaging analysis. The difference in peak ratios could be due to the isotopically emitted photons that are more likely to be scattered away from 
(a)

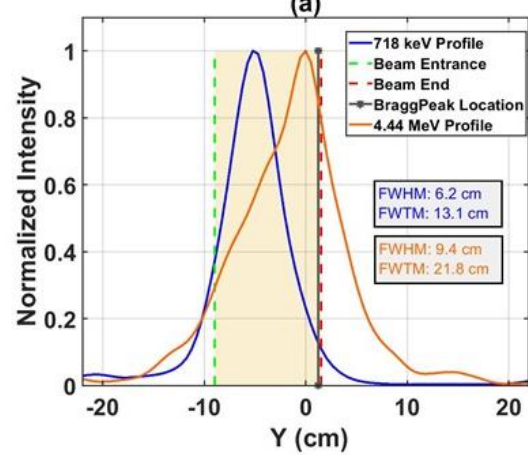

(c)

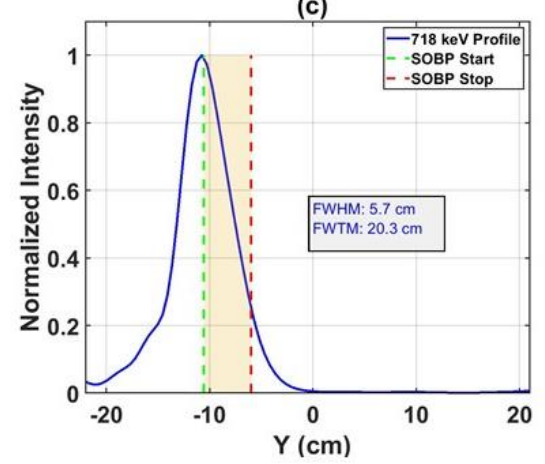

(b)

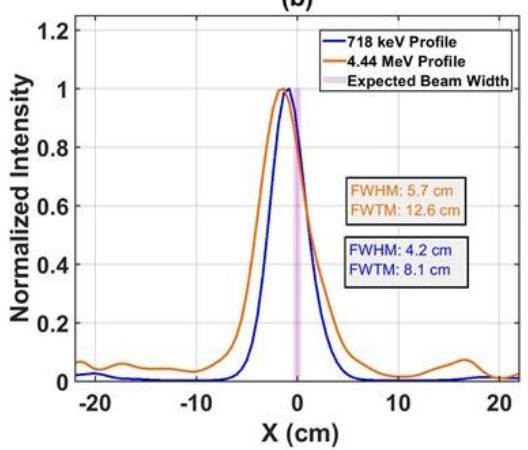

(d)

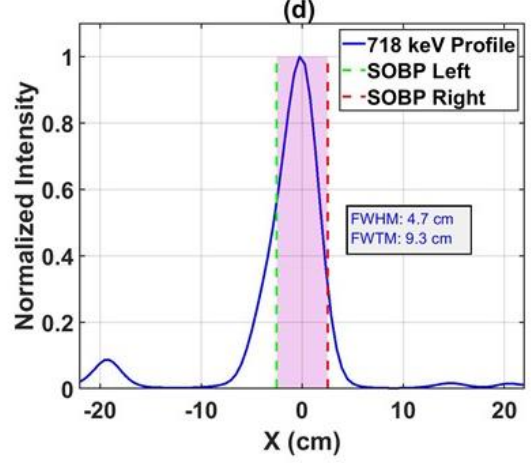

Fig. 5. FWHM and FWTM Uncertainty Analysis. (a) $1 \mathrm{D}$ profiles of the $718 \mathrm{keV}$ and the $4.44 \mathrm{MeV}^{\mathrm{Angle}} 270^{\circ}$ image reconstructions along the beam trajectory. (b) $1 \mathrm{D}$ profiles of the $718 \mathrm{keV}$ and $4.44 \mathrm{MeV}$ angle $270^{\circ}$ image reconstructions in comparison to the expected beam width from SRIM simulations (3.8 mm width). (c) $1 \mathrm{D}$ profile of the $718 \mathrm{keV} \mathrm{SOBP} 5 \mathrm{~cm}^{3}$ image reconstruction along the beam trajectory in comparison to the expected location SOBP region of $5 \mathrm{~cm}$ in the y direction. (d) $1 \mathrm{D}$ profile of the $718 \mathrm{keV} \mathrm{SOBP} 5 \mathrm{~cm}^{3}$ image reconstruction in the lateral direction in comparison to the expected location of the SOBP region of $5 \mathrm{~cm}$ in the $\mathrm{x}$ direction. All FWHM and FWTM measurements result in a $+/-0.5 \mathrm{~cm}$ error based on pixel resolution.

the detector when the beam is not parallel to the detector, resulting in a reduction in the number of photons recorded.

For each image reconstruction, one can note the noise pattern that is constant for each measurement due to the inherent noise from background, down-scatter, partial energy deposition events, and missequencing of events during image reconstruction. The Angle $270^{\circ}$ measurement results in the best image reconstruction of both the $718 \mathrm{keV}$ and $4.44 \mathrm{MeV}$ photopeaks which could be in-part due to the angle of the beam trajectory.

The $718 \mathrm{keV}$ photopeak is the most prominent energy line from the carbon capture emissions seen in the spectra presented in both experiments. However, the $4.44 \mathrm{MeV}$ photopeak is dampened by Polaris J3 worsened energy resolution at higher energies, but also due to the doppler broadening expected from this prompt gamma emission. For this peak both experiments show a very broad structure of the single and double escape peaks and a slight feature around $4.44 \mathrm{MeV}$ in the spectral results. This is reflected in the image reconstructions, which results in poorer reconstruction of the hotspot.

The images illustrate that the MLEM approach for Compton imaging can give a reliable estimate of the beam's location within the target. This is contingent on the number of recorded photopeak events and the ability to suppress those events that reconstruct as noise in the image space. Still, work needs to be done to validate the reconstructed hotspots location to determine the accuracy of the image reconstruction with respect to defining the Bragg peak location within millimeter resolution. Although the presented images show reconstructions within the beam range, the pixel resolution $(0.5$ $\mathrm{cm}^{2}$ ) used for reconstruction is not fine enough to resolve an estimate of this magnitude.

This work concludes that the large volume CZT detector used, Polaris J3, is capable of recording both sub and super 1 $\mathrm{MeV}$ prompt gammas for analysis of proton beam characterization. The image reconstructions of the $718 \mathrm{keV}$ line from carbon, shows that when the beam's trajectory is parallel to the detection system, the hotspot is more prominent and is not hindered by the noise in the background. The $4.44 \mathrm{MeV}$ reconstruction in this trajectory also shows that the detection system can be used to estimate the endpoint of the beam, given enough statistical counts in the photopeak, despite the poorer energy resolution.

There is much room for improvement for the detection system used. Currently the dynamic range is only calibrated for up to $3 \mathrm{MeV}$ and each module within the detector records events independently. Thus, interplanar events are not leveraged as of yet, which current research by H3D has shown improvement in high energy photon imaging by utilizing interplanar events in digital CZT systems [23]. In these measurements the detector does see some paralyzation, due to such a high flux of photons but is still able to achieve definitive spectral results and informative imaging results at both high beam doses and energies.

The uncertainty analysis based on the FWHM and FWTM of the image reconstructions is limited by the imaging resolution. 
A pixel pitch of $0.5 \mathrm{~cm}$ is too large to pinpoint the exact location of the hotspot reconstructions. To achieve submillimeter resolution an alternative approach to imaging the data must be used to allow for high-resolution imaging without being computationally limited. However, despite the resolution limits the FWHM analysis shows that both photopeaks used for reconstruction fall with in range of the proton beam as expected and can provide information on the shape and location of the beam, the $4.44 \mathrm{MeV}$ lending more info to the Bragg peak location.

\section{ACKNOWLEDGEMENTS}

The authors of this work would like to thank Professor Emeritus Ronald Fleming for his invaluable insight and contribution to this work and the team of reviewers of this work for their thoughtful suggestions and comments. The research reported in this publication was supported by the National Institutes of Health National Cancer Institute under award number R01CA187416. The content is solely the responsibility of the authors and does not necessarily represent the official views of the National Institutes of Health.

\section{REFERENCES}

[1] H. Liu, and J. Y. Chang, "Proton therapy in clinical practice," Chinese Journal of Cancer, vol. 30, no. 5, pp. 315-326, 2011.

[2] D. Schardt, T. Elsässer, and D. Schulz-Ertner, "Heavy-ion tumor therapy: Physical and radiobiological benefits," Rev. Mod. Phys., vol. 82, pp. 383425,2010

[3] J. Krimmer, D. Dauvergne, J. M. Letang, and E. Testa, "Prompt-gamma monitoring in hadrontherapy: A review," Nucl Intstr Met Phys Res A, vol. 878, pp. 58-73, https://dx.doi.org/10.1016/j.nima.2017.07.063, 2018.

[4] K. Parodi and J. C. Polf, "In vivo range verification in particle therapy," Med Phys, vol. 45, pp. e1036-e1050, 10.1002/mp.12960, Nov 2018.

[5] P. Solevi, et al., "Performance of MACACO Compton telescope for ionbeam therapy monitoring: first test with proton beams," Physics in Medicine \& Biology, vol. 61, pp. 5149-5165.2016

[6] F. H. González, M. Rabe, T. Ruggieri, T. Bortfeld, J. M. Verburg, “A fullscale clinical prototype for proton range verification using prompt gamma-ray spectroscopy," Physics in Medicine \& Biology, vol. 63, 2018.

[7] E. Draeger, et al. "3D prompt gamma imaging for proton beam range verification," Physics in Medicine \& Biology, vol. 63, no. 3, 13pp, 2018.

[8] J.C. Polf, et al. "Imaging of prompt gamma rays emitted during delivery of clinical proton beams with a Compton camera: feasibility studies for range verification," Physics in Medicine \& Biology, vol. 60, pp. 7085$7099,2015$.

[9] B. Kozlovsky, R. J. Murphy, and R. Ramaty, "Nuclear Deexcitation Gamma-Ray Lines from Accelerated Particle Interactions," The Astrophysical Journal Supplement Series, vol. 141 no. 2, pp. 523-541, 2002.

[10] P. G. Ortega et al., "Noise evaluation of Compton camera imaging for proton therapy," Physics in Medicine \& Biology, vol. 60, no. 5, pp. 1845, 2015.

[11] Panthi R, Maggi P, Peterson S, Mackin DM, Polf JC, and Beddar S, "Secondary particle interactions in a Compton camera designed for in vivo range verification of proton therapy," IEEE Trans Radiat Plasma Med Sci, vol. 4, pp. 1-1, DOI: 10.1109/TRPMS.2020.3030166, 2021.

[12] J. S. Loeffler, M. Durante, "Charged particle therapy-optimization, challenges and future directions," Nature Reviews Clinical Oncology, vol. 10, pp. 411-424, 2013.

[13] J. C. Polf, S. Peterson, G. Ciangaru, M. Gillin, and S. Beddar, "Prompt gamma-ray emission from biological tissues during proton irradiation: a preliminary study," Physics in Medicine and Biology, vol. 54, no. 3, pp. 731-743, 2009.

[14] B. Gottschalk, "Radiotherapy proton interactions in matter." arXiV:1804.00022v1 [physics.med-ph], 2018.
[15] Z. He, W. Li, G. F. Knoll, D. K. Wehe, J. Berry, and C. M. Stahle, "3-D position sensitive CdZnTe gamma-ray spectrometers," Nucl. Inst. \& Meth. A, vol. 422, pp. 173-8, 1999.

[16] J. Ziegler, J. P. Biersack, M. D. Ziegler, SRIM: The Stopping and Range of Ions in Matter, SRIM Co., 2015.

[17] K. Rusiecka, et al., "Determination of Gamma Angular Distribution from the Shape of Spectral Line for the First Excited State of Carbon Nucleus," World Journal of Nuclear Science and Technology, 06. 63-70. 10.4236/wjnst.2016.61006. 2016.

[18] A. P. Dempster, N. M. Laird, and D. B. Rubin, "Maximum likelihood from incomplete data via the EM algorithm," J. Royal Statist. Soc., vol. B39, pp. 1-38, 1977.

[19] C. E. Lehner, Z. He, F. Zhang, "4pi Compton Imaging Using a 3-D Position-Sensitive CdZnTe Detector Via Weighted List-Mode Maximum Likelihood," IEEE Trans. Nucl. Sci., vol. 51, no. 4, pp. 1618-1624, 2004.

[20] J. Kim, et al., "Charge sharing in common-grid pixelated CdZnTe detectors," Nucl. Instr. \& Meth. A, vol. 654, no.1, pp 233-243, 2011.

[21] D. Shy, Z. He, "Gamma-Ray Tracking for High-Energy Gamma-Ray Imaging in Pixelated CdZnTe," Nuclear Inst. and Methods in Physics Research, 2018.

[22] D. Shy, J. Xia, Z. He, "Artifacts in high-energy Compton imaging with 3D position sensitive CdZnTe," IEEE Transactions of Nuclear Science, 2020.

[23] H. H. Chen-Mayer, S. Brown, H. Yang, "Feasibility study of Compton imaging for PGAA," Journal of Radioanalytical and Nuclear Chemistry, vol. 322, pp. 1729-1738. 2019. 